\title{
Fabry-Perot method for the characterization of integrated optical directional couplers
}

\author{
C. Vázquez, P. Baquero, and F. Hernández-Gil
}

\begin{abstract}
A method to measure the loss and the power-transfer ratio of directional couplers is presented. It is based on the Fabry-Perot resonances from end-facet reflections. The dependence of the accuracy of the measurements on the facet tilts is described. For low facet misalignments, an uncertainty $<0.3 \mathrm{~dB}$ in the loss measurements and power-transfer-ratio measurement errors $<1 \%$ are obtained. For arbitrary facet tilts the errors depend on the extinction ratios. Experimental measurements are reported to verify the method, and its application to multimode interference couplers is shown.

Key words: Integrated optical directional couplers, characterization, Fabry-Perot effects. (1) 1995 Optical Society of America
\end{abstract}

\section{Introduction}

Optical couplers are key components in integrated optics, both for signal routing and signal processing. The availability of a good technique to confirm that the fabricated coupler exhibits characteristics as designed is very important.

Different methods have been proposed to measure the coupling coefficient, ${ }^{1,2}$ whereas the coupler loss is usually measured relativetostraight reference guides fabricated on the same chip. Most of the measure ment methods currently used are difficult to apply because they require a very careful alignment of the samples in the optical benches, and it is difficult to obtain good accuracy and repeatability. Besides, the measurements to be taken are strongly affected by reflections from the interfaces between the waveguides and the input and output media (frequently air). ${ }^{3}$ These effects are particularly severe for semiconductor waveguides, because of their high refractive indices (typically approximately 3.2). FabryPerot $(\mathrm{FP})$ resonances as functions of the wavelength of the input beam are generated by these end-facet reflections. So, depending on the length of theinputoutput guides, different measurements of the loss and the coupling coefficient are obtained. These effects have been used for loss measurement on waveguides, ${ }^{4}$ y junctions, ${ }^{5}$ and directional couplers ${ }^{3}$ for highextinction-ratio devices.

In this paper, we present a method for measuring the power-transfer ratio $\mathrm{K}_{r}$ and the loss $\mathrm{G}$ based on $\mathrm{a}$ FP technique. We consider the influence of facet misalignments on measurements of coupled-waveguide devices. We show that our FP method can be used to measure overall coupler loss with an uncertainty of $<0.3 \mathrm{~dB}$ for low facet misalignments. Concerning power-transfer-ratio measurements, the error is $<1 \%$ under the same conditions. Measurements of $K_{r}$ and $G$ are shown to confirm the validity of the method.

When arbitrary facet tilts are considered, loss measurements with an uncertainty $<0.8 \mathrm{~dB}$ are obtained for $90 \%$ of the values of the unknown phase shifts for devices with low extinction ratios $(<6 \mathrm{~dB})$, with an upper-limit loss error $<2 \mathrm{~dB}$ (lower error bounds than those obtained by Tomlinson et al) ${ }^{3}$. So, concerning the loss measurement of couplers with unknown facet tilts, both methods are complementary. For low extinction ratios our method will provide lower errors, and for high extinction ratios the Tomlinson technique will bemore accurate.

\section{Theory}

In this section we describe the model that is used for the directional coupler and the assumptions that have been made in deriving the theoretical expressions. We also summarize the basic theoretical results. 


\section{A. Assumptions and Definitions}

The directional-coupler model is illustrated in Fig. 1. It is assumed that the access waveguides support only a single guided mode. The reflecting facets at the input and output guides are not exactly normal to the guides, so there are phase shifts of $\Delta \Phi_{1}$ and $\Delta \Phi_{2}$ for light propagating in the input and output guide pairs, respectively. It is assumed that the two input guides' facets have the same (amplitude) reflectivity $r_{1}$ and the two output guides' facets have the same reflectivity $r_{2}$ for light in the guided mode. The (amplitude) transmissions of the facets for light in the waveguide mode to the external field are $\mathrm{tf}_{1}$ for the input facets and $\mathrm{tf}_{2}$ for the output facets. These values are not required in the $F P$ technique.

Light propagating in any of the guides 2 and 3 experiences a total optical phase shift $\Phi_{1}=\beta_{1} l_{1}$ and $\Phi_{2}=\beta_{2} l_{2}$, where $\beta_{1}$ and $\beta_{2}$ are the propagating constants of the guided mode of guides 2 and 3 , respectively. $I_{1}$ is the physical length of guide 2 , and $\mathrm{I}_{2}$ is the length of guide 3 . The guides 1 and 4 experience a total optical phase shift $\Phi_{1}+\Delta \Phi_{1}$ with $\Delta \Phi_{1}=\beta_{1} \Delta \mathrm{I}_{1}$ and $\Phi_{2}+\Delta \Phi_{2}$ with $\Delta \Phi_{2}=\beta_{2} \Delta \mathrm{I}_{2}$, where $\Delta \mathrm{I}_{1}$ and $\Delta \mathrm{I}_{2}$ are the length differences between guides 1 and 2 and guides 3 and 4 , respectively. The (amplitude) transmission is $t_{1}$ for the input guides and $t_{2}$ for the output guides. These transmission factors include not only the propagation loss of the guides, but also any additional loss resulting from bends.

\section{B. Derivation of Transmission Equations}

To calculate the transmitted optical fields, we simply multiply together the various transmission factors to determine the contribution to the output from each successive round-trip of the light.

We can write a set of $2 \times 2$ transmission matrices that describe the action of the various parts of the coupler on the field vectors (see Fig. 1). After multiplying them in the proper order and adding all the contributions to the output fields $E_{3}$ and $E_{4}$, the following expressions are derived ${ }^{3}$ :

$$
\begin{aligned}
\mathrm{E}_{3}= & \frac{\left(1-\mathrm{m}_{22}\right) \mathrm{A}+\mathrm{m}_{12} \exp \left(\mathrm{i} \Delta \Phi_{2}\right) \mathrm{B}}{\left(1-\mathrm{m}_{11}\right)\left(1-\mathrm{m}_{22}\right)-\mathrm{m}_{12} \mathrm{~m}_{21}} \\
& \times \mathrm{tf}_{1} \mathrm{tf}_{2} \mathrm{t}_{1} \mathrm{t}_{2} \exp (\mathrm{i} \Phi) \exp \left(\mathrm{i} \Delta \Phi_{1}\right) \mathrm{E}_{1}, \\
\mathrm{E}_{4}= & \frac{\mathrm{m}_{21} \mathrm{~A}+\left(1-\mathrm{m}_{11}\right) \exp \left(\mathrm{i} \Delta \Phi_{2}\right) \mathrm{B}}{\left(1-\mathrm{m}_{11}\right)\left(1-\mathrm{m}_{22}\right)-\mathrm{m}_{12} \mathrm{~m}_{21}} \\
& \times \mathrm{tf}_{1} \mathrm{tf}_{2} \mathrm{t}_{1} \mathrm{t}_{2} \exp (\mathrm{i} \Phi) \exp \left(\mathrm{i} \Delta \Phi_{1}\right) \mathrm{E}_{1},
\end{aligned}
$$

with $T=r_{1} r_{2} t_{1}^{2} t_{2}^{2}$ and $\Phi=\Phi_{1}+\Phi_{2}$.

The coupler is modeled by a symmetric $2 \times 2$ matrix:

$$
H=\left[\begin{array}{ll}
A & B \\
B & A
\end{array}\right],
$$

where $A$ and $B$ depend on the type of coupler. An input field $E_{1}$ in guide 1 has been considered.

C. Output Powers for Reflecting Facets Normal to the Guides

The output powers $\left|E_{3}\right|^{2}$ and $\left|E_{4}\right|^{2}$ are derived with Eqs. (1) for reflecting facets normal to the guides, so $\Delta \Phi_{1}=\Delta \Phi_{2}=0$.

An ideal coupler is going to be considered, so the elements of the matrix $\mathrm{H}$ have the form

$$
\begin{aligned}
& A=\left[g\left(1-K_{r}\right)\right]^{1 / 2}, \\
& B=\sqrt{\mathbf{g K}_{r}} \exp [i(\pi / 2)],
\end{aligned}
$$

where $g$ is the fractional loss of the coupler without the transmission loss of the input-output guides

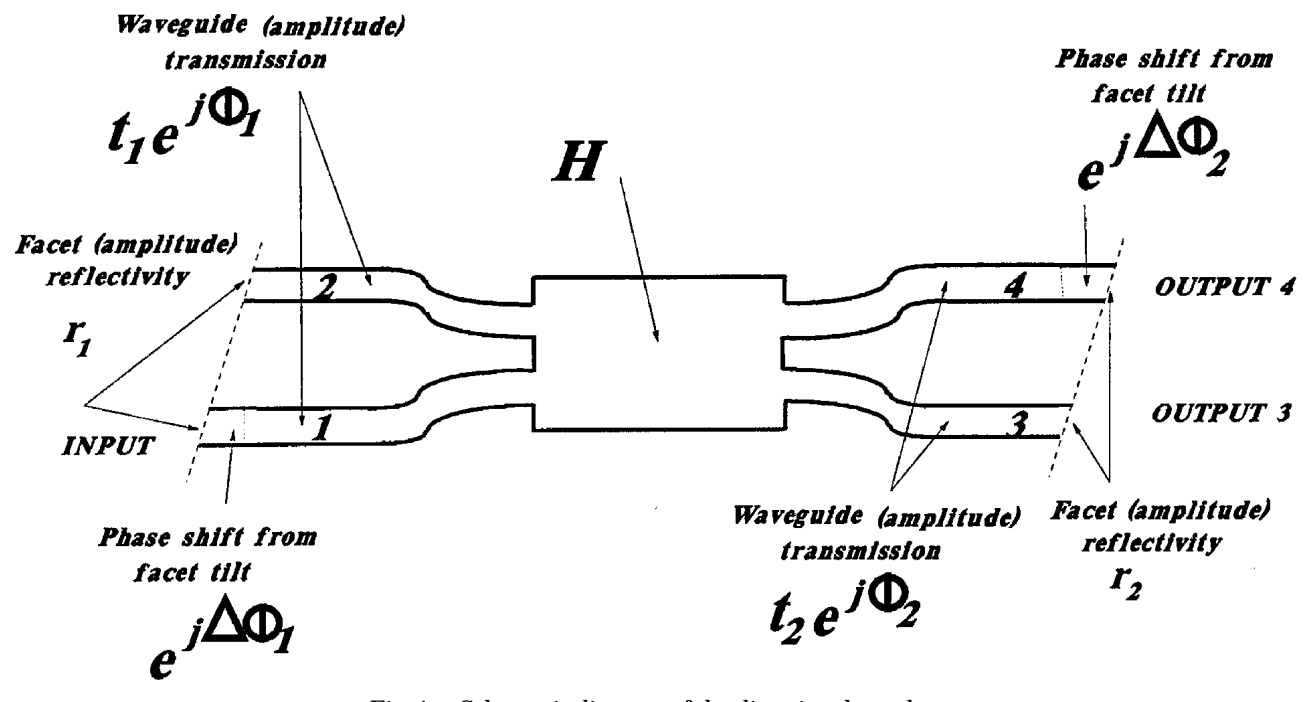

Fig. 1. Schematic diagram of the directional coupler. 
being taken into consideration and $\mathrm{K}_{\mathrm{r}}$ is the powertransfer ratio.

Substituting Eq. (2) into Eq. (1), we show that the output powers aregiven by $r_{1} r_{2}=0$ is the other parameter we are going to measure.

Output powers $\mathrm{P}_{3}$ and $\mathrm{P}_{4}$ are periodic functions of the angle $\theta$. If we consider a total length $I=I_{1}+I_{2}$

$$
\begin{aligned}
& \mathrm{P}_{3}(\Theta)=\frac{\left(1-\mathrm{F}_{1} \cos \theta\right) \mathrm{tf}_{1}{ }^{2} \mathrm{tf}_{2}{ }^{2} \mathrm{t}_{1}{ }^{2} \mathrm{t}_{2}{ }^{2} \frac{|\mathrm{A}|^{2}}{1+|\mathrm{C}|^{2}} \mathrm{P}_{1}}{\left[1+\mathrm{F}_{1}{ }^{2}\left(1-2 \mathrm{~K}_{\mathrm{r}}\right)^{2}\right]-2 \mathrm{~F}_{1}\left(2 \mathrm{~K}_{\mathrm{r}}-1\right) \cos \Theta-\mathrm{F}_{1}{ }^{2} \sin ^{2} \Theta}, \\
& \mathrm{P}_{4}(\theta)=\frac{\left(1+\mathrm{F}_{1} \cos \theta\right) \mathrm{tf}_{1}{ }^{2} \mathrm{tf}_{2}{ }^{2} \mathrm{t}_{1}{ }^{2} \mathrm{t}_{2}{ }^{2} \frac{|\mathrm{B}|^{2}}{1+|\mathrm{C}|^{2}} \mathrm{P}_{1}}{\left[1+\mathrm{F}_{1}{ }^{2}\left(1-2 \mathrm{~K}_{\mathrm{r}}\right)^{2}\right]-2 \mathrm{~F}_{1}\left(2 \mathrm{~K}_{\mathrm{r}}-1\right) \cos \Theta-\mathrm{F}_{1}{ }^{2} \sin ^{2} \Theta},
\end{aligned}
$$

where $C=T\left(A^{2}-B^{2}\right) \exp (i \theta)=T G \exp (i \theta), F_{1}=$ $2 \mathrm{C} \mid /\left(1+|\mathrm{C}|^{2}\right) \mathrm{y} \Theta=2 \Phi$. $\quad \mathrm{P}_{1}$ is the input power at port 1. The total intrinsic loss $G$ is defined by $G=$ $\left(P_{3}+P_{4}\right) / P_{1}$ in the case of $r_{1}=r_{2}=0$.

This is the loss value we want to measure. The power-transfer ratio defined by $\mathrm{P}_{4} /\left(\mathrm{P}_{3}+\mathrm{P}_{4}\right)$ for

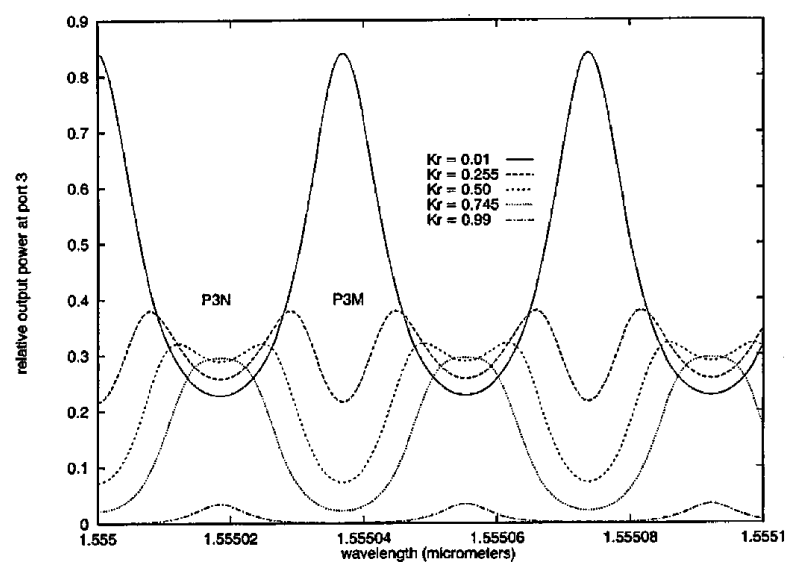

(a)

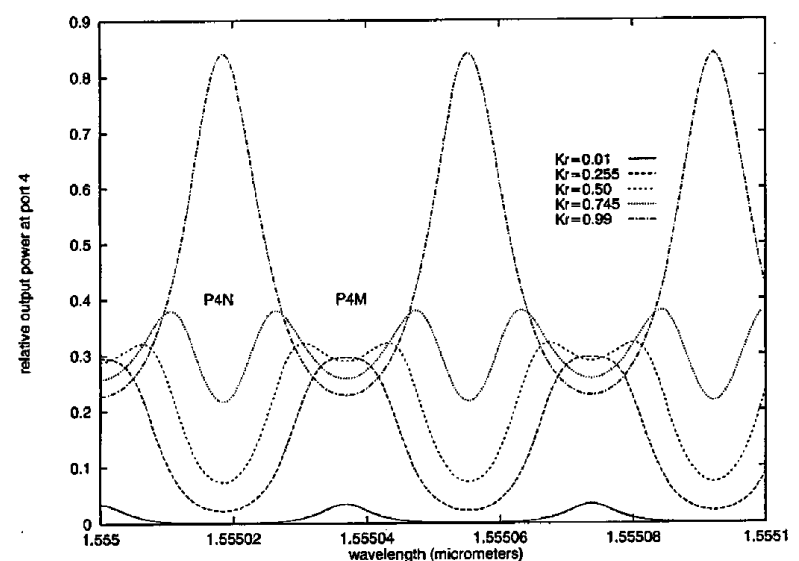

(b)

Fig. 2. Calculated output power at (a) port $3, \mathrm{P}_{3}$, and (b) port $4, \mathrm{P}_{4}$, for a symmetric coupler as a function of the wavelength, with tilt facets normal to the guides, for $G=0.9, r_{1} r_{2}=0.35$, and for various values of $\mathrm{K}_{\mathrm{r}}$. and $\mathrm{N}$ is the propagation index of the fundamental mode of the input-output waveguides, the angle $\theta$ can be written as $\theta=2[(2 \pi N I) / \lambda]$. So by varying the wavelength of the input beam $\lambda$, we can modify $\theta$.

In the following, we analyze the dependence of the profiles of the output powers on the parameters $G$ and $\mathrm{K}_{\mathrm{r}}$.

Figure 2 shows the relative output powers at ports 3 and $4, P_{3}$ and $P_{4}$, respectively, as a function of the wavelength for a coupler with $\mathrm{G}=0.9(0.45 \mathrm{~dB})$ and different $K_{r}$ values. $r_{1} r_{2}=0.35$ (a typical value for InP waveguides) with reflecting facets normal to the input-output guides. We have considered $\mathrm{N}=3.268$ and $\mathrm{I}=1 \mathrm{~cm}$.

As was expected, when $K_{r}=1$, in port 3 there are no $F P$ resonances because all the power is transferred to guide 4 . The device acts as a single waveguide. ${ }^{4}$

Depending on the $K_{r}$ value, one or two maxima appear in each period. A maximum for a certain $\mathrm{K}_{\mathrm{r}}$ value can turn to a relative or even absolute mi nimum for another $\mathrm{K}_{r}$ value (see the points labeled by $\mathrm{P}_{3 \mathrm{~N}}$ in Fig. 2). For a certain $K_{r}$ value, if we analyze the output-power profiles, thereis no change from maxima to minima. Only deeper FP resonances appear for low-loss couplers.

\section{Measurement Methods}

To achieve a measurement method insensitive to experimental conditions, we use ratios between measured powers.

In the following we describe three different methods based on the FP resonances of the coupler.

\section{A. Numerical Calculation Procedure}

The device characteristics are obtained from the foll owing equations system:

$$
\begin{aligned}
& t_{3}=\frac{P_{3 \max }}{P_{3 \min }}=\Omega_{1}\left(K_{r}, G\right), \\
& t_{4}=\frac{P_{4 \max }}{P_{4 \min }}=\Omega_{2}\left(K_{r}, G\right),
\end{aligned}
$$


where $\mathrm{P}_{3 \max }, \mathrm{P}_{3 \min }, \mathrm{P}_{4 \max }, \mathrm{P}_{4 \min }$ are the maximum and minimum output power at ports 3 and 4 and $\Omega_{1}$ and $\Omega_{2}$ are derived from Eqs. (3).

Figures $3(\mathrm{a})$ and $3(\mathrm{~b})$ show contour plots of the functions $\Omega_{1}\left(K_{r}, G\right)$ and $\Omega_{2}\left(K_{r}, G\right)$, respectively, as functions of the power-transfer ratio $K_{r}$ and the loss $G$ for a coupler with a facet reflectivity of $r_{1} r_{2}=0.35$.

The possible combination of values of $K_{r}$ and $G$ that correspond to given measured values of $t_{3}$ and $t_{4}$ is determined with Fig. 3. It is clear that there are cases in which two solutions appear, but they corre-

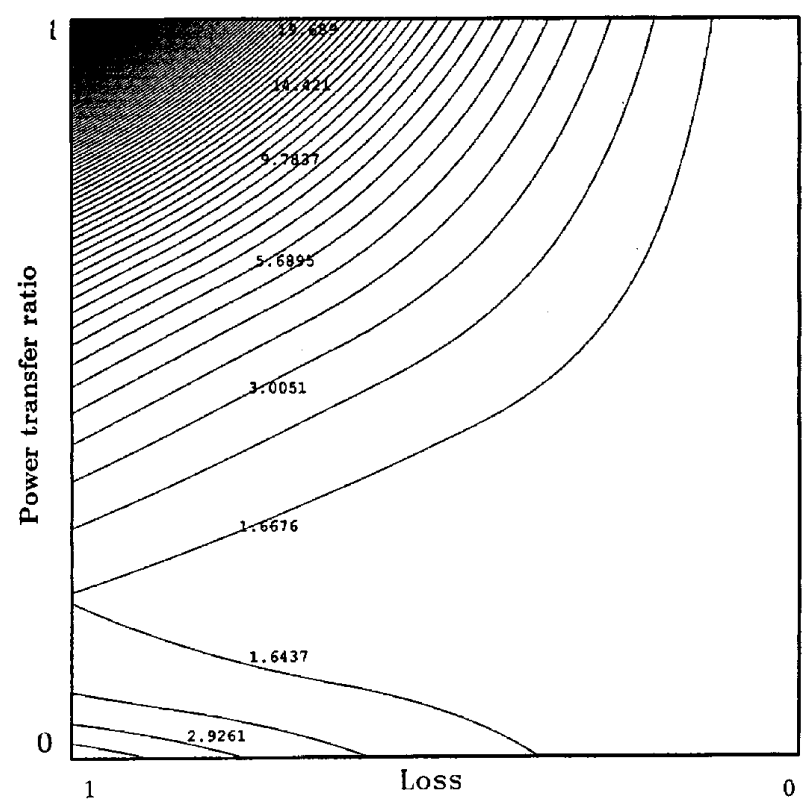

(a)

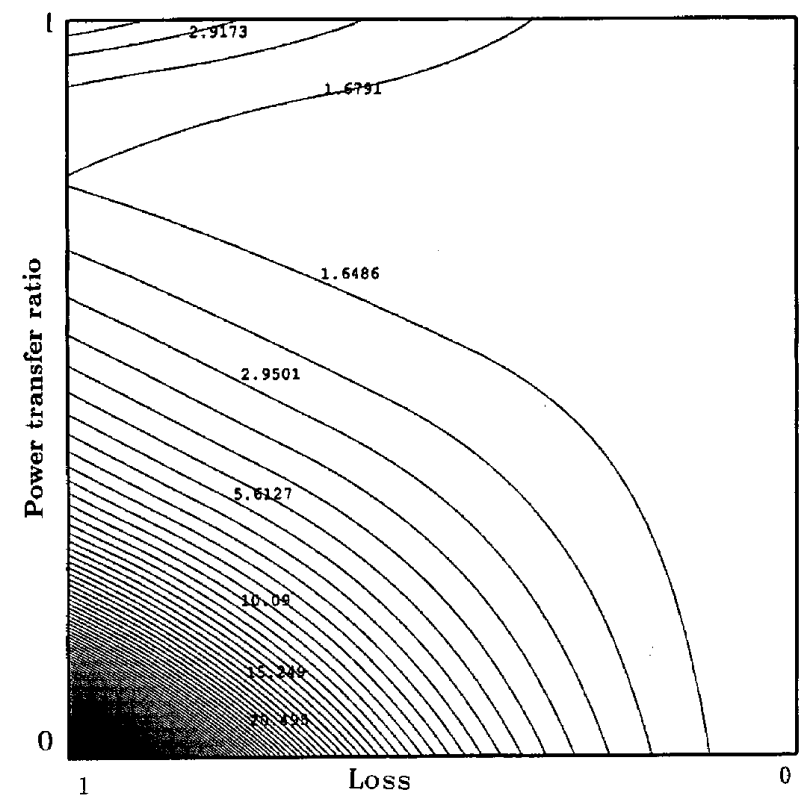

(b)

Fig. 3. Contour plots of the ratios $(a) t_{3}$ and $(b) t_{4}$ as functions of the coupling coefficient $\left(\mathrm{K}_{\mathrm{r}}\right)$ and the loss $(\mathrm{G})$ for couplers with a facet reflectivity of $r_{1} r_{2}=0.35$. spond to different output-power profiles as functions of the wavelength. Only one of them would be similar to the spectral-response measurements, as can be seen in the measurements reported in Section 5. The intersection points shown in Figs. $3(a)$ and $3(b)$ are symmetric about the line $K_{r}=1 / 2$, as was expected. From this intersection one can also determine that for $\mathrm{K}_{\mathrm{r}}<1 / 2$ it follows that $\mathrm{t}_{3}<\mathrm{t}_{4}$, and for $\mathrm{K}_{\mathrm{r}}$ $>1 / 2$ it follows that $t_{3}>t_{4}$.

\section{B. Analytical Calculation Procedure}

As a first step in determining an equations system with an analytic solution for $K_{r}$ and $G$, we try to find the maxima and minima of the output powers. So we derive Eqs. (3) with respect to $\theta$. These derivatives are equal to zero in any of the following cases:

$$
\begin{aligned}
\operatorname{sen} \theta= & 0 \rightarrow \cos \theta=\left\{\begin{array}{ll}
+1 & P_{3}=P_{3 M}, P_{4}=P_{4 M} \\
-1 & P_{3}=P_{3 N}, P_{4}=P_{4 N}
\end{array},\right. \\
\cos \theta= & \left\{\frac{2}{F_{1}} \pm 2^{3 / 2}\left[1-2 F_{1}^{2}\left(1-K_{r}\right)\right.\right. \\
& \left.\left.+2 F_{1}^{2}\left(1-K_{r}\right)^{2}-\left(1-2 K_{r}\right)\right]^{1 / 2}\right\} \mid\left(F_{1} / 2\right) .
\end{aligned}
$$

When we use Eq. (5) and substitute in Eqs. (3), the value of the output powers will be

$$
\begin{aligned}
\mathrm{P}_{3 \mathrm{M}[3 \mathrm{~N}]}= & \mathrm{tf}_{1}^{2} \mathrm{tf}_{2}^{2} \frac{|\mathrm{A}|^{2}}{1+|\mathrm{C}|^{2}} \\
& \times \frac{1-[+] \mathrm{F}_{1}}{\left[1+\mathrm{F}_{1}^{2}\left(1-2 \mathrm{~K}_{\mathrm{r}}\right)^{2}\right]-[+] 2 \mathrm{~F}_{1}\left(1-2 \mathrm{~K}_{\mathrm{r}}\right)}, \\
\mathrm{P}_{4 \mathrm{M}[\mathrm{AN}]}= & \mathrm{tf}_{1} \mathrm{tff}_{2}^{2} \frac{|\mathrm{B}|^{2}}{1+|\mathrm{C}|^{2}} \\
& \times \frac{1+[-] \mathrm{F}_{1}}{\left[1+\mathrm{F}_{1}{ }^{2}\left(1-2 \mathrm{~K}_{\mathrm{r}}\right)^{2}\right]-[+] 2 \mathrm{~F}_{1}\left(1-2 \mathrm{~K}_{\mathrm{r}}\right)},
\end{aligned}
$$

where the different parameters $A, C, F_{1}, t_{1}, t_{2}$ have been defined in Section 2.

The brackets notation is used to point out the sign change depending on the considered extreme $\mathrm{M}$ or $\mathrm{N}$ in each port.

In deriving an equation for $\mathrm{G}$, we must find expressions independent of $K_{r}$, so we define $s=\left[\left(P_{3 M} P_{4 N}\right) /\right.$ $\left.\left(P_{3 N} P_{4 M}\right)\right]^{1 / 2}$, and operating with Eqs. (7), we obtain

$$
\mathrm{G}=\frac{1}{\mathrm{r}_{1} \mathrm{r}_{2}} \frac{1-\sqrt{\mathrm{S}}}{1+\sqrt{\mathrm{s}}}
$$

If we now define the ratio $r=P_{3 M} / P_{3 N}$, the resulting equation for $0 \leq K_{r} \leq 1$ after we operate 
with Eqs. (7) is

$$
1-K_{r}=\frac{1}{2}+\frac{(\sqrt{r}-\sqrt{s})^{2}}{2(r-s)} \frac{1+s}{1-s} .
$$

The extremes $P_{3 M}, P_{3 N}, P_{4 M}$, and $P_{4 N}$ can be seen in Fig. 2. They are usually relativeand absoluteminima if two maxima appear in each period, otherwise the relative minimum is a maximum.

To identify the extremes easily when one is measuring the output powers, a deeper analysis of Eqs. (7) is carried out.

We first consider $K_{r}<1 / 2$, which happens for $\mathrm{t}_{3} / \mathrm{t}_{4}<1$.

Now, when we suppose that $P_{4 M}>P_{4 N}$, using Eq. (7), we obtain

$$
0<2 \mathrm{~F}_{1}\left(1+2 \mathrm{x}+\mathrm{x}^{2} \mathrm{~F}_{1}^{2}\right) .
$$

Equation (10) is valid for any $0<\mathrm{K}_{\mathrm{r}}<0.5$ value that makes $x=\left(1-2 K_{r}\right)>0$. This implies that our supposition is al ways followed, so $\mathrm{P}_{4 \mathrm{~N}}$ is the absolute minimum and $P_{4 M}$ is the relative minimum.

Following Eq. (5), we show that $P_{3 M}$ is the extreme that appears at the same wavelength as $P_{4 M}$. But when measuring the output powers, we modify the injection current of the laser, and the same injection current does not have to mean the same wavel ength. So it should be desirable to have another way to identify the extremes at port 4.

If we consider $P_{3 M}<P_{3 N}$, using Eq. (7), we obtain

$$
0<2 \mathrm{~F}_{1}\left(1-2 \mathrm{x}+\mathrm{x}^{2} \mathrm{~F}_{1}^{2}\right) \text {. }
$$

The values of $K_{r}$ that follow Eq. (11) are $K_{\lim }<$ $\mathrm{K}_{\mathrm{r}}<0.5$, where $\mathrm{K}_{\lim }=1 / 2\left[1-\mathrm{F}_{1^{2}}{ }^{2}\left[1-\left(1-\mathrm{F}_{1}{ }^{2}\right)^{1 / 2}\right]\right.$. This power-transfer ratio limit varies from 0.2 to 0.25 depending on $\mathrm{G}$. So if $\mathrm{K}_{\mathrm{r}}<\mathrm{K}_{\lim }<0.5$, then $\mathrm{P}_{3 \mathrm{~N}}$ is the absolute minimum, and for $0.5>K_{r}>K_{\lim }$ is $P_{3 M}$ the absolute minimum.

If we swap the value of $P_{3 M}$ for $P_{3 N}$, an absurd result is obtained for $0 \leq K_{r} \leq 1$ or $0 \leq \mathrm{G} \leq 1$ when Eqs. (8) and (9) are applied, unless we are dealing with values of $K_{r}$ close to $K_{\text {lim. }}$ In that case, the numerical calculation procedure should be used to obtain the right solution.

An equivalent analysis can be developed for $K_{r} \geq$ $1 / 2$. It is depicted in Fig. 4, in which the extreme is the absolute minimum in each port for any $G$ and $K_{r}$ values.

Both calculation procedures have been derived for analysis of symmetric couplers. If a small asymmetry is considered, Eqs. (8) and (9) are still valid for calculation of the values of $G$ and $K_{r}$ (seetheAppendix). At the output-power profiles of nonsymmetric couplers, two maxima with different amplitudes $\left(\mathrm{P}_{\max _{1}}, \mathrm{P}_{\max _{2}}\right)$ appear, as revealed in the simulations. So in the numerical calculation procedure, a maximumaverageintensity $P_{\max }=\left(P_{\max _{1}}+P_{\max _{2}}\right) / 2$, should be used at each port.

Multimode interference (MMI) couplers can be also characterized by applying Eqs. (8) and (9), as is
Identification of the extremes

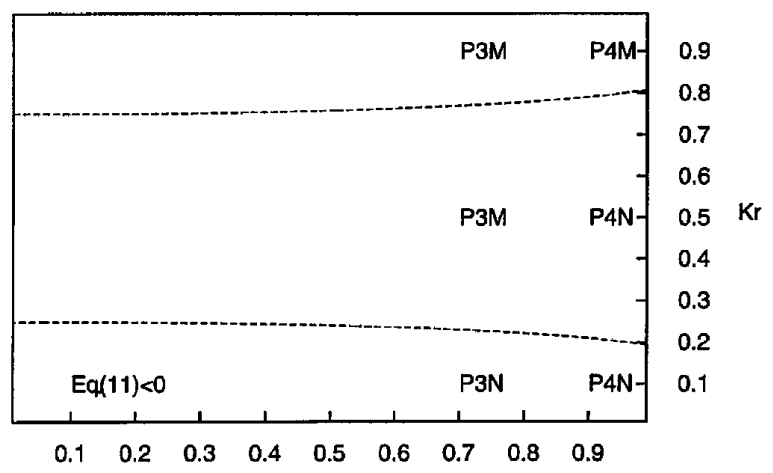

G

Fig. 4. Contour plot of expression (11), $0<2 \mathrm{~F}_{1}\left(1-2 x+x^{2} \mathrm{~F}_{1}{ }^{2}\right)$ with $\mathrm{G}$ and $\mathrm{K}_{\mathrm{r}}$ as parameters. The absolute minimum at each port is identified.

demonstrated in the Appendix. A model of the coupler based on the self-image property of multimode waveguides described in detail by Ulrich and $\mathrm{Ka}$ maya ${ }^{6}$ is considered. It is also corroborated with a multimodal analysis.

C. Tomlinson Technique

Tomlinson ${ }^{3}$ reported another technique for characterizing directional couplers based on scanned measurements. His technique was not based on proposing an exact solution of an equations system, but on defining independent quantities that are easy to measure. He defined an extinction ratio $R_{\text {scan }}$ :

$$
R_{\text {scan }}=10 \log \left(\frac{P_{3 \max }+P_{3 \min }}{P_{4 \max }+P_{4 \min }}\right) d B .
$$

Although the loss was defined as $\mathrm{G}_{\text {scan }}=10 \mathrm{log}$ $\left(T_{\exp } / r_{1} r_{2}\right)$, with the factor $T_{\exp }$ given by

$$
T_{\text {exp }}=\frac{1-\left(1-F^{2}\right)^{1 / 2}}{F}, \quad F=\frac{P_{\text {max }}-P_{\min }}{P_{\text {max }}+P_{\min }} .
$$

This is the equation for measuring the loss of a single waveguide.

Tomlinson applied Eq. (13) to the data of the output port with the larger average intensity $\left(\mathrm{P}_{\max }+\mathrm{P}_{\min }\right) / 2$.

\section{Influence of the Phase Shifts from Facet Tilts}

Equations ( 8 ) and (9) are exact solutions for measuring $\mathrm{K}_{\mathrm{r}}$ and $\mathrm{G}$ in the case of reflecting facets normal to the waveguides. But if this is not the case, the profiles of the output powers can be modified by the facet misalignments. The effect is fairly similar to that of the asymmetry: two maxima of different amplitude appear.

\section{A. Estimation of the Facet Misalignments}

Chiefly it can be considered that the path difference comes from alignment problems. A realistic estima- 
tion in our fabrication process should be to assume a vertical displacement of $2 \mu \mathrm{m}$ in alignment of samples that have a $2-\mathrm{cm}$ separation, which means a maximum $\Delta \Phi_{1}= \pm 0.064$ rad for $50-\mu \mathrm{m}$ input-output waveguide separation, $\mathrm{N}=3.268$ and $\lambda=1.55 \mu \mathrm{m}$. Both cuts on the sample are made following the crystallographic planes of the crystal (which should mean $\Delta \Phi_{2}=\Delta \Phi_{1}$ because they should be parallel cuts).

On the other hand, because of low shear strain in a perfect lattice, we should write down $\Delta \Phi_{2}=\Delta \Phi_{1}+$ $\Delta \Phi$. In a semiconductor material with high purity, $10^{4}-10^{5}$ dislocations $/ \mathrm{cm}^{2}$ are typical values. If a 50- $\mu \mathrm{m}$ branch separation and a 0.85- $\mu \mathrm{m}$ thickness are considered, $\Delta \Phi= \pm 0.0014 \mathrm{rad}$ is the maximum deviation. But the presence of a certain number of piled-up dislocations (i.e., 1000 dislocations $/ 50 \mu \mathrm{m}$ ) implies $\Delta \Phi= \pm 2 \pi$, if a 25 - $\AA$ dislocation size is taken into account, so arbitrary phase shifts should be considered.

These rough calculations have been made to justify the sel ection of the two cases that are analyzed bel ow. We study the maximum $\mathrm{K}_{\mathrm{r}}$ and $\mathrm{G}$ measurement errors when applying our analytical calculation procedure and the Tomlinsom technique for

$$
\Delta \Phi_{1}=\Delta \Phi_{2} \leq \pm 0.064 \mathrm{rad}
$$

$\Delta \Phi_{2}=\Delta \Phi_{1}+\Delta \Phi$, arbitrary phase shifts $\Delta \Phi_{1}$ and $\Delta \Phi$ from 0 to $2 \pi$.

\section{B. Evaluation of the Error}

To evaluate the maximum possible measurement errors, we define the power-transfer-ratio error and the loss error:

$$
\begin{gathered}
\Delta K_{r}=K_{r, \exp }-K_{r, 0}, \\
\Delta G=10 \log \left(\frac{G_{\exp }}{G_{0}}\right) .
\end{gathered}
$$

The subscript 0 is related to the intrinsic values, which means that $r_{1} r_{2}=0$. These intrinsic values as functions of $A$ and $B$ have the form

$$
\begin{aligned}
\mathrm{K}_{\mathrm{r}, 0} & =\frac{|\mathrm{B}|^{2}}{|\mathrm{~A}|^{2}+|\mathrm{B}|^{2}}, \\
\mathrm{G}_{0} & =10 \log \left[\mathrm{t}_{1}{ }^{2} \mathrm{t}_{2}^{2}\left(|\mathrm{~A}|^{2}+|\mathrm{B}|^{2}\right)\right] .
\end{aligned}
$$

$\mathrm{K}_{\mathrm{r}, \exp }$ and $\mathrm{G}_{\exp }$ correspond to the values of the power-transfer ratio and the loss measured with any of the measurement methods. The output powers are calculated for the corresponding values of $\Delta \Phi_{1}$ and $\Delta \Phi_{2}$ with Eqs. (1).

When we are interested in estimating the error related to our analytical calculation procedure, Eqs. (8) and (9) are applied together with Eqs. (14).

In case we want to estimate the error obtained in using the Tomlinson technique, Eqs. (12) and (13) are used together with Eqs. (14).

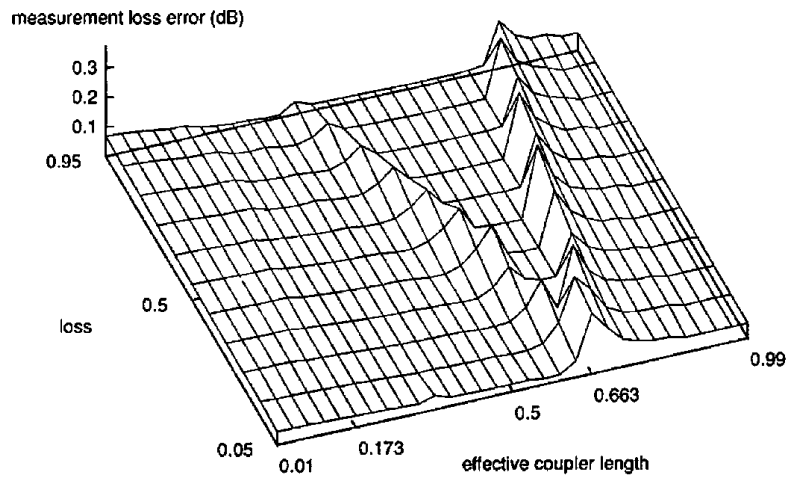

Fig. 5. Surface plots of the maximum measurement loss error using the analytic calculation procedure described in this paper, as functions of the normalized effective coupling length $\left(L_{c} / \pi\right)$ and loss $(\mathrm{G})$ for phase shifts $\Delta \Phi_{1}=\Delta \Phi_{2} \leq \pm 0.064 \mathrm{rad}$ and a facet reflectivity of $r_{1} r_{2}=0.35$.

In the following calculation we have assumed that $r_{1} r_{2}=0.35$.

\section{Symmetric Couplers}

Figure 5 shows a surface plot of the maximum loss-measurement error $\Delta G_{\max }$ as a function of normalized effective coupler length and intrinsic loss for phase shifts $\Delta \Phi_{1}=\Delta \Phi_{2} \leq \pm 0.064$ rad for a symmetric coupler.

This normalized effective coupler length $L_{c} / \pi$ is defined in the Appendix, and it is related to the power-transfer ratio: $\mathrm{K}_{\mathrm{r}}=\sin ^{2}\left(\mathrm{~L}_{c} / 2\right)$ if $\gamma=1$.

This $\Delta G_{\max }$ is less than $0.3 \mathrm{~dB}$ for any values of $K_{r}$ and $G$. Furthermore, $\Delta G_{\max }$ is less than $0.1 \mathrm{~dB}$ for a coupler with $\mathrm{K}_{\mathrm{r}} \leq 0.5$.

The higher errors come from the effect of the facet misalignments. In Fig. 6 output powers are depicted for data where this problem appears. One can see that $P_{4 N}$ is al tered if the phase shift $\Delta \Phi_{1}$ increases, so instead of detecting $P_{4 N}$ 's values for $\Delta \Phi_{1}=0$, we detect the closest maximum.

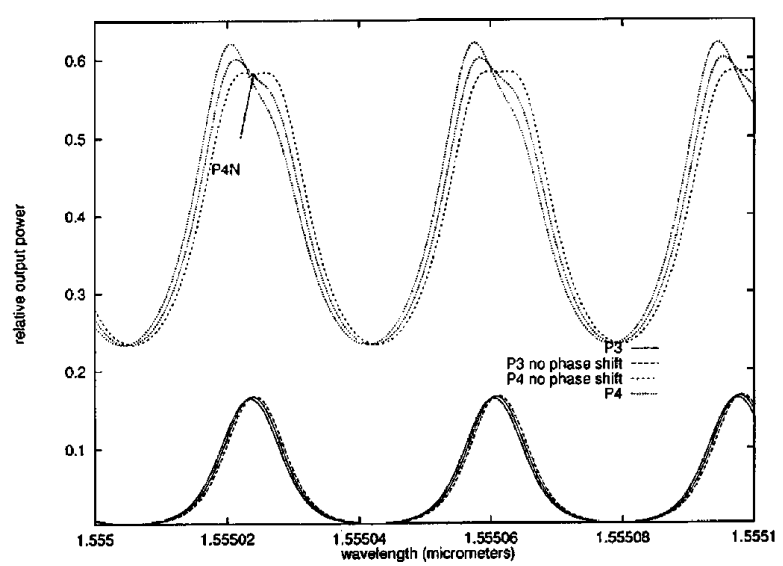

Fig. 6. Relative output power as a function of the wavelength for a coupler with $L_{c} / \pi=0.833$ and $G=0.95$. 
When the Tomlinson technique is applied, the errors are less than $0.3 \mathrm{~dB}$ only for power-transfer ratios $\mathrm{Kr} \geq 0.01$ for $\mathrm{K}_{\mathrm{r}} \leq 0.99$. The values that make the coupler behave as a single waveguide are the particular case from which Tomlinson has derived his loss-measurement equation.

If $\mathrm{K}_{\mathrm{r}} \sim 0.5$ the Tomlinson technique also reports very low errors, but they risesteeply to $10-14 \mathrm{~dB}$ as $\mathrm{K}_{\mathrm{r}}$ departs from this value.

The maximum power-transfer-ratio measurement error is less than $1 \%$ except for the cases in which the output power depicted in Fig. 6 appears. But this is a local effect, which is easy to identify when measure ments are made and has a small probability.

With the Tomlinson technique, the power-transfer ratio depends slightly on the intrinsic loss, but in any case it reports higher errors than our method. If arbitrary facet misalignments are considered, the errors increase.

For low extinction ratios $<6 \mathrm{~dB}\left(0.8>\mathrm{K}_{\mathrm{r}}>0.2\right)$ couplers, the loss-error range obtained with our technique is at least a factor of 3 smaller than with the Tomlinson one (where there is a bumpy plateau at $\sim 10 \mathrm{~dB}$.

The low probability of the higher error bounds ( $\Delta \mathrm{G}<0.8 \mathrm{~dB}$ in the $90 \%$ values of the phase shifts) is an interesting property of our cal culation procedure.
These calculations are for an intrinsic loss $\mathrm{G}_{0}=4.96$ $\mathrm{dB}$, but the error bounds are not very dependent on $\mathrm{G}_{0}$.

\section{Nonsymmetric Couplers}

A description of a nonsymmetric coupler is given in the Appendix. $\quad \gamma$ describes the degree of asymmetry of the coupler.

$\Delta \mathrm{G}_{\max }$ has been calculated for $\Delta \Phi_{1}=\Delta \Phi_{2} \leq \pm 0.064$ $\mathrm{rad}, \mathrm{G}_{0}=0.25(6 \mathrm{~dB})$ as a function of the asymmetry $\gamma$ and the power-transfer ratio. $\Delta \mathrm{G}_{\max }$ is $\leq 0.25 \mathrm{~dB}$ for $\gamma=1 \pm 0.05$. Within that range, the maximum error in $\mathrm{K}_{\mathrm{r}}$ is less than $1 \%$.

When arbitrary facet misalignments are involved, the above-mentioned probability of having $\Delta G<0.8$ $\mathrm{dB}$ is getting worse for nonsymmetric couplers. For instance, if $\gamma=0.9$ in only $75 \%$ of the values of the phase shifts, $\Delta \mathrm{G}<0.8 \mathrm{~dB}$. These calculations are again for an intrinsic loss $\mathrm{G}_{0}=4.96 \mathrm{~dB}$.

\section{Experimental Results}

Parallel waveguide couplers were fabricated on InP with a ridge waveguide structure, which was achieved by the use of the reactive ion etching process with a $\mathrm{CH}_{4} / \mathrm{H}_{2}$ mixture within RF-assisted plasma. The waveguide of the measured device was excited by the use of a distributed feedback 1.55- $\mu \mathrm{m}$ laser at TE

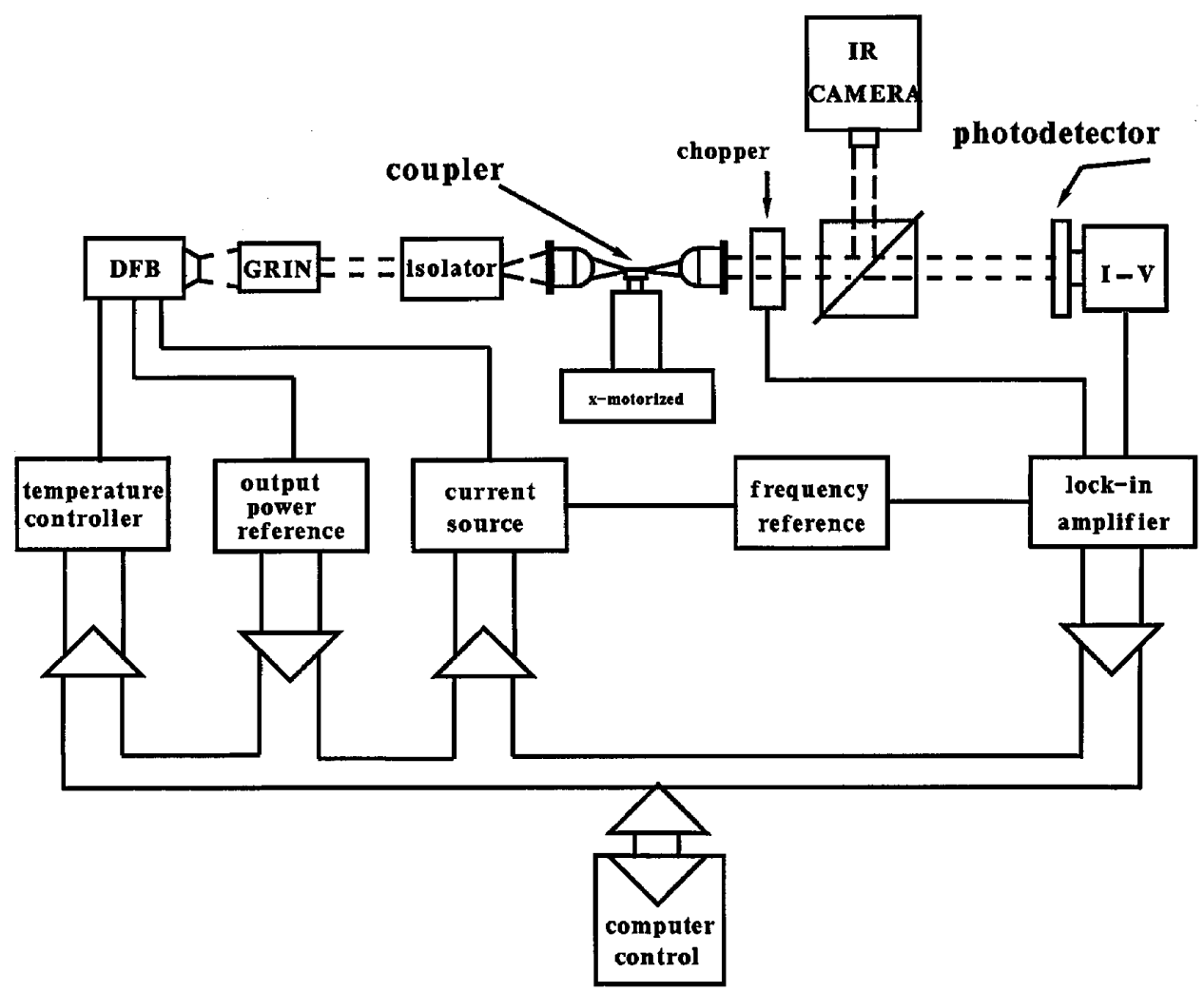

Fig. 7. Setup for characterization of optical directional couplers by the use of theFP method. DFB, distributed feedback; GRIN, gradient index. 
polarization with a microscope objective lens. The laser spectral linewidth was less than $60 \mathrm{MHz}$, so no loss penalty related to the linewidth requirements appeared.7 The output light from the waveguide was focused with another microscope objective. A beam splitter diverted part of the beam to a TV monitor and the rest to a photodetector. To discriminate between the beam coming from the waveguide and any other reflected light, a chopper was inserted between the objective lens and the beam splitter. The experimental setup used in the measurements is depicted in Fig. 7.

The value of $r_{1} r_{2}=0.35$ was experimentally estimated with waveguides from the same sample. This was done by successi vely cleaving the guide to shorter lengths and measuring the loss, assuming that the successive cleaves give approximately equal facet reflectivities. ${ }^{4}$

Measurements of waveguide loss with the FP method $^{4}$ by the use of temperature variation and wavelength variation were taken, and results with a relative error of $1 \%$ were obtained. Waveguide loss $<0.2 \mathrm{~dB} / \mathrm{cm}$ was obtained, another indicator of the good quality of the material. The wavelength varia-

[PORT 4]

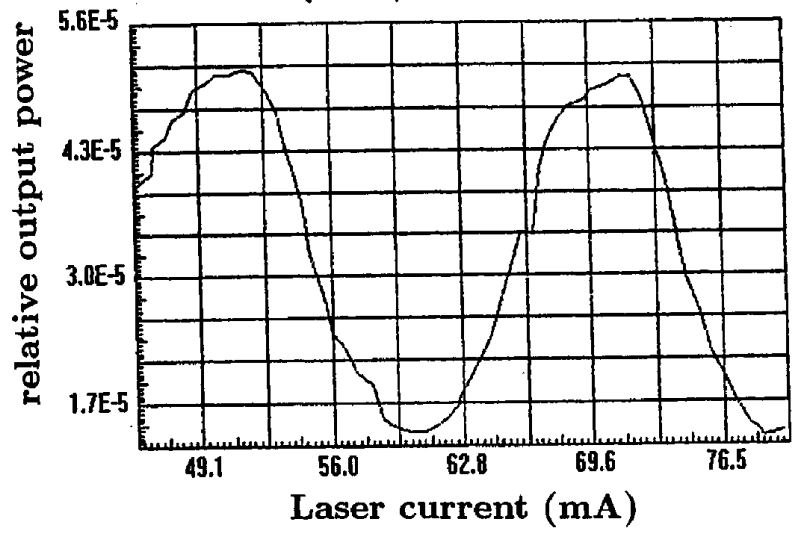

[PORT 3]

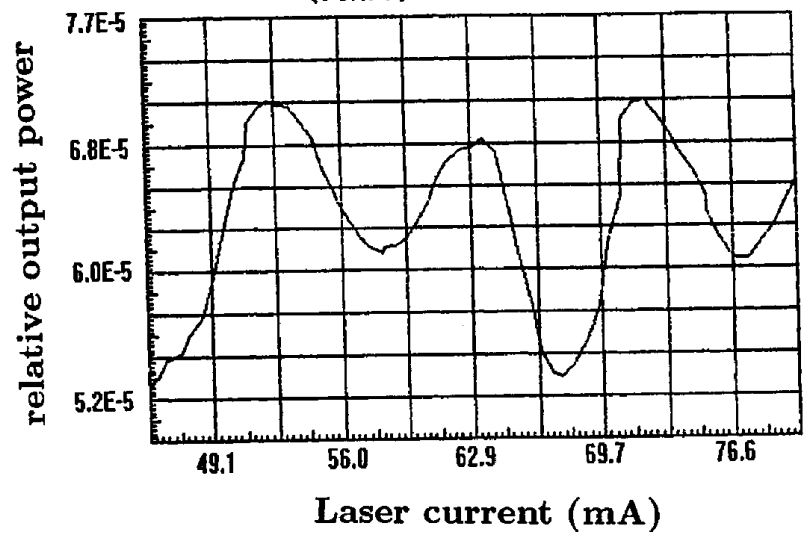

Fig. 8. Measured relative output power at ports $3\left(\mathrm{P}_{3}\right)$ and $4\left(\mathrm{P}_{4}\right)$ as a function of the laser injection current (in milliamperes) for a parallel waveguide coupler.

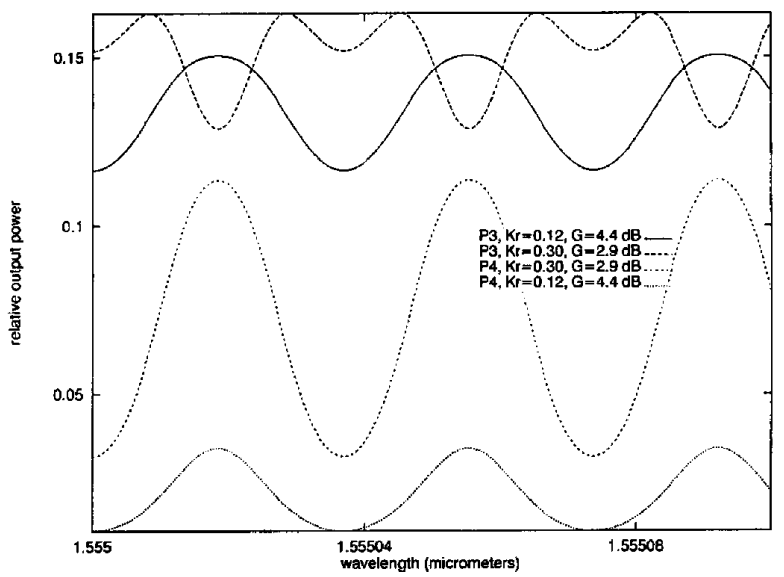

Fig. 9. Calculated output power at ports 3 and 4 as functions of the wavelength of couplers with $\Delta \Phi_{1}=\Delta \Phi=0, r_{1} r_{2}=0.35$ and (long- and short-dashed curves) $\mathrm{K}_{\mathrm{r}}=0.30, \mathrm{G}=2.9 \mathrm{~dB}$ and (solid and dotted curves) $\mathrm{K}_{\mathrm{r}}=0.12, \mathrm{G}=4.4 \mathrm{~dB}$.

tion consists of varying the injection current of the temperature-stabilized laser. Figure 8 shows the FP resonance measurements for both outputs. At port 3 , there are two maxima with a slight difference in amplitude, which means that $\Delta \Phi_{1} \neq 0$ or $\gamma \neq 1$, or both. Anyway, because of the dimensions of the coupler and the quality of the material, the difference in amplitude of both maxima is more likely to be the result of $\Delta \Phi_{1}$.

\section{A. Uniqueness of Solutions}

We apply our numerical calculation procedure, with the measurements of Fig. 8 and the curves of Fig. 3, to determine the value of $\mathrm{K}_{r}$ and $\mathrm{G}$. There are two possible solutions, and the output-power profiles for each of them are plotted in Fig. 9. After comparing Figs. 8 and Fig. 9, it can be seen that $K_{r}=0.3, G=$ $2.88 \mathrm{~dB}$ is the uniquesolution. Applying our analytical calculation procedure, we obtain $\mathrm{K}_{\mathrm{r}}=0.3$ and $\mathrm{G}=$ $3 \mathrm{~dB}$, in perfect concordance with the previous results.

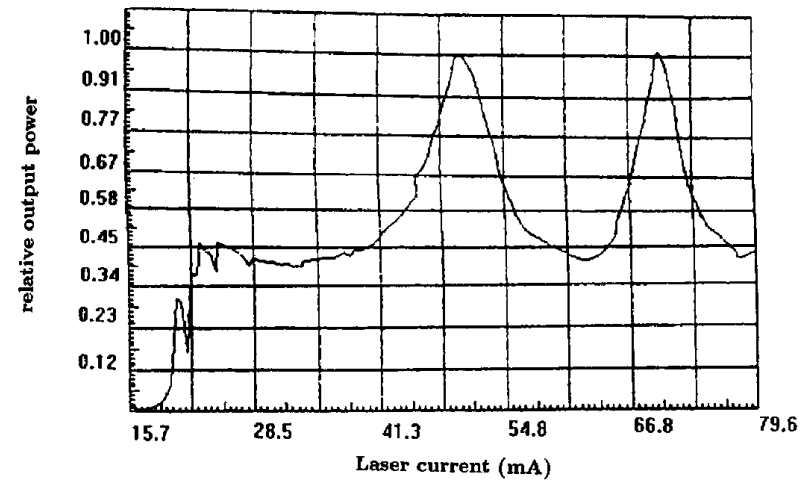

Fig. 10. Measured relative output power at port 3 as a function of the laser injection current (in milliamperes) for a parallel waveguide coupler. 
Because of the accuracy of alignment and cleaving and the lack of dislocations, the estimations of Section 4 are considered, so $\Delta \Phi_{1}=\Delta \Phi_{2} \leq \pm 0.064 \mathrm{rad}$.

In those conditions, the expected errors in the measurements from phase shifts are $\mathrm{K}_{\mathrm{r}}=0.3 \pm 0.001$, $\mathrm{G}=2.88 \pm 0.005 \mathrm{~dB}$.

B. Contrast between Measurements with and without Considering the Fabry-Perot Resonances

Figure 10 shows the power transmission in the device as a function of the laser injection current for a typical directional coupler. FP resonances can be observed for currents between 40 and $80 \mathrm{~mA}$.

If laser injection currents (I) near the laser threshold are used, these resonances disappear because of the low coherence of the signal light $(1<25 \mathrm{~mA}$ at Fig. 10). Measurements of the power-transfer ratio for different injections currents I $<17 \mathrm{~mA}$ weretaken simply by measuring the two output powers. Less than $2 \%$ deviation in the measurements was obtained.

Figure 11 shows the results of measuring the power-transfer ratio in eight couplers fabricated in the same chip. These couplers are in the same sample as the one previously measured, so the qual ity of the material and the characteristics of the fabrication process are the same.

The power-transfer-ratio measurements with the numerical calculation procedure, the analytic calculation procedure (both based on the FP resonances) and the laser biased at low current $(1<17 \mathrm{~mA})$ to achieve a low coherence signal are shown in Fig. 11 for each coupler.

It can be seen that the measurements obtained with the two calculation procedures proposed in this paper are slightly different, less than $3 \%$, in some couplers. This is mainly because when the reflecting facets are not normal to the waveguide axis, both calculation procedures are different approximations.

When both FP resonance measurements are compared with the non-FP measurements, the deviation is also very small, which confirms our hypothesis of

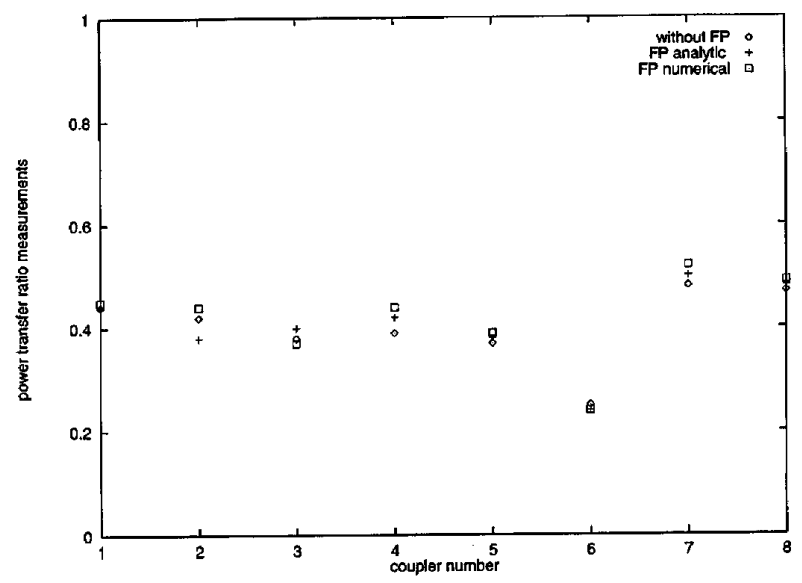

Fig. 11. Power-transfer-ratio measurements.
$\Delta \Phi_{1}=\Delta \Phi_{2} \leq \pm 0.064 \mathrm{rad}$, because otherwise the expected error should be higher.

\section{Conclusions}

In this paper, two calculation procedures based on the use of the FP resonances to calculate the powertransfer ratio and the loss of directional couplers were shown.

For good-quality materials and a precise fabrication process, the reflecting facets are nearly normal to the input and output guides, and the errors expected are very low. The uncertainty when the loss is measured is $<0.3 \mathrm{~dB}$ for symmetric couplers. The power-transfer ratio uncertainty is $<1 \%$. But in case there is no information about the facet misalignments, the uncertainty introduced when the powertransfer ratio is measured increases. For lowextinction-ratio $(<6 \mathrm{~dB})$ couplers, measurements of loss with the analytical calculation procedure have an uncertainty at least 3 times smaller than the one reported by Tomlinson in other scanned measurements. Error loss $<0.8 \mathrm{~dB}$ is obtained for $90 \%$ of the values of the unknown phase shifts because of facet misalignments. For high-extinction-ratio $(>15 \mathrm{~dB})$ couplers, the Tomlinson technique reports lower loss errors. In this way it can be said that both techniques are complementary for measuring the loss of directional couplers with arbitrary facet misalignments.

Measurements of the power-transfer ratios of eight couplers under the condition of facets nearly normal to the waveguides have been reported. Measurements obtained with our calculation procedures and those obtained with a more conventional technique that avoids FP resonances were compared. Deviations of less than 3\% in the measurements of the power-transfer ratio with the different methods have been obtained.

The FP method should be adequate to characterize multimode interference couplers and could be modified for characterizing devices with a higher number of input and output ports.

\section{Appendix A.}

We now apply our FP calculation procedures to nonsymmetric and multimode interference couplers.

\section{A. Nonsymmetric Couplers}

In calculating Eqs. (3) we have considered an ideal coupler with a phase shift of $\pi / 2$ between both output guides.

For nonsymmetric couplers this property is not fulfilled, so we rename $C=r_{1} r_{2}\left(A^{2}-B^{2}\right) \exp (i \theta)=$ $C \mid \exp \left(i \delta_{C}\right)$ and $D=r_{1} r_{2}\left(A^{2}+B^{2}\right) \exp (i \theta)=|D| \exp \left(i \delta_{D}\right)$, where

$$
\delta_{\mathrm{D}}=\delta_{\mathrm{C}}+\xi,
$$

so $\xi$ is a measurement of the asymmetry of the coupler. 
Operating as we did in Subsection 3.B., we show that the transmitted output power will be given by of the coupler. If $L_{c}=n \pi$, where $n$ is an integer, the coupler is in the bar state when $\mathrm{n}$ is even and in the

$$
\begin{aligned}
& \left(1-F_{1} \cos \theta\right) \mathrm{tf}_{1}^{2} \mathrm{tf}_{2}^{2} \frac{|\mathrm{A}|^{2}}{1+|\mathrm{C}|^{2}} \\
& P_{3}(\theta)=\frac{\left(1+F_{1}^{2}\left|\frac{D}{C}\right|^{2}\right)-\cos \xi\left[2 F_{1}\left|\frac{D}{C}\right|\right] \cos \theta-F_{1}^{2} \sin ^{2} \theta-\sin \xi F_{1}^{2}\left(\frac{D}{|C|}\right)^{2} \frac{\left(1-|C|^{2}\right)}{|D|} \sin \theta}{(, .,} \\
& \left(1+F_{1} \cos \theta\right) \mathrm{tf}_{1}^{2} \mathrm{tf}_{2}^{2} \frac{|\mathrm{B}|^{2}}{1+|\mathrm{C}|^{2}}
\end{aligned}
$$

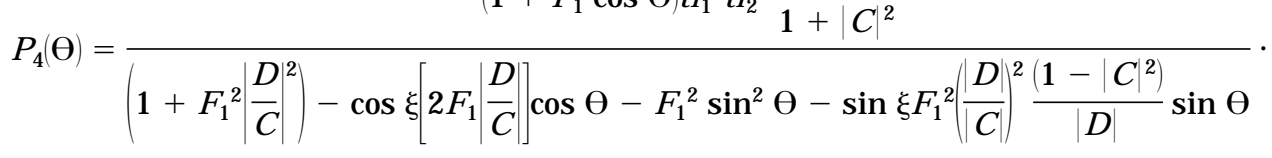

Comparing E qs. (3) and (A2), one can see that a new term in $\sin \theta$ appears in the denominator, so the extreme condition $\sin \theta=0$ is only fulfilled if

$$
\sin \xi \mathrm{F}_{1}{ }^{2} \frac{|\mathrm{D}|^{3}\left(1-|C|^{2}\right)}{|C|^{2}}=0 .
$$

Equation (8) was obtained from ratios of power, avoiding the presence of denominators, so this new term will not affect the loss equation if the condition (A3) is foll lowed.

There is al so a factor in $\cos \xi$ in the denomi nators of Eqs. (2), which implies a dependence on $\xi$ in the recalculated power-transfer-ratio equation:

$$
1-K_{r}=\frac{1}{2}+\frac{(\sqrt{r}-\sqrt{s})^{2}}{2(r-s)} \frac{(1+s) \cos \xi}{1-s} .
$$

Knowing the degree of asymmetry, we can exactly determine $K_{r}$. The problem can be inverted, so we could measure $\xi$ from $\mathrm{K}_{\mathrm{r}}$.

In the limit, for $\gamma \rightarrow 1$ then $\xi \rightarrow 0$, so $\cos \xi \rightarrow 1$ and Eqs. (A3)-(A4) are equivalent to Eqs. (8) and (9).

We are going to calculate $\xi$ for a two-mode-couplingregion coupler. The coefficients $A$ and $B$ of the matrix $\mathrm{H}$ that defines the coupler are given by ${ }^{3}$

$$
\begin{aligned}
\mathrm{A} & =\mathrm{t}_{\mathrm{s}} \eta_{\mathrm{s}}{ }^{2} \exp \left(i \Phi_{c}\right) \Psi_{+} \text {with } \\
\Psi_{+} & =\frac{\exp \left(i \mathrm{~L}_{c} / 2\right)+\gamma \exp \left(-\mathrm{i} \mathrm{L}_{c} / 2\right),}{2} \\
\mathrm{~B} & =\mathrm{t}_{\mathrm{s}} \eta_{\mathrm{s}}{ }^{2} \exp \left(i \Phi_{c}\right) \Psi_{-} \text {with } \\
\Psi_{-} & =\frac{\exp \left(i \mathrm{~L}_{c} / 2\right)-\gamma \exp \left(-\mathrm{iL} \mathrm{L}_{c} / 2\right)}{2},
\end{aligned}
$$

where $L_{c}=\left(\beta_{s}-\beta_{a}\right) l_{c}, I_{c}$ being the effective physical length of the coupler and $\beta_{s}$ and $\beta_{a}$ the propagation constants of the symmetric and antisymmetric modes of the coupler, respectively. Note that although $L_{c}$ is referred to as an effective coupler length, it has the dimensions of a phase shift. $L_{c}$ defines the behavior cross state when $n$ is odd. For the case of $L_{c}=$ $(n+1 / 2) \pi$ we have a 3-dB coupler. $\Phi_{c}=\left(\beta_{s}+\beta_{a}\right) I_{c} / 2$ is the average phase shift of the coupler. The degree of asymmetry of the coupler is defined as $\gamma=$ $t_{a} \eta_{a}{ }^{2} t_{s} \eta_{s}{ }^{2}$, where $t_{s}, t_{a}$ are the amplitude transmissions of the symmetric and antisymmetric modes of the coupler, respectively, and $\eta_{\mathrm{s}}, \eta_{\mathrm{a}}$ are the efficiencies of the symmetric and antisymmetric modes at the input of the coupler, respectively. Trying to find an equivalence with the ideal coupler in Subsection 2.C., we have for the symmetric case $\gamma=1$, so $\Psi_{+}=$ $\cos \left(L_{c} / 2\right)=\left(1-K_{r}\right)^{1 / 2}$ and $\Psi_{-}=\exp (i \pi / 2) \sin \left(L_{c} / 2\right)=$ $\exp (\mathrm{i} \pi / 2) \sqrt{\mathrm{K}_{r}}$.

Substituting the values of $A$ and $B$ given by Eqs. (A5) into Eqs. (A1), we find out that

$$
\xi=\arctan \left[\frac{\left(1-\gamma^{2}\right) \sin L_{c}}{\left(1+\gamma^{2}\right) \cos L_{c}}\right] .
$$

\section{B. Multimode Interference Couplers}

Multimode interference (MMI) couplers are less sensitive to deviation fabrications, ${ }^{8}$ so they are becoming very attractive in many applications.

The output field $E(x)$ at the image plane when the input is excited at $x_{0}$ by a delta function is given by ${ }^{6}$

$$
E(x)=2 \sum_{m=0}^{M} \frac{\tau_{m}}{W_{a}} \cos \left(k_{m} x\right) \cos \left(k_{m} x_{0}\right),
$$

where $\tau_{m}, W_{a}$, and $k_{m}$ are the transfer coefficient, the width of the region of power flow, and the transverse spatial frequency of each mode, respectively; $M$ is the highest guided mode. We define $A=E\left(x_{0}\right), B=$ $\mathrm{E}\left(\mathrm{W}-\mathrm{x}_{0}\right)$, where $\mathrm{W}$ denotes the width of the multimode region.

It is going to be considered that $x_{0}=W / 3$ (so the modes $2,5, \ldots$ will not be excited). $\tau_{m}=\tau_{m} \mid \exp \left(j \beta_{m}\right)$ where $\beta_{m}$ are the propagation constants of each mode and $L_{M M I}$ is the length of the MMI region. $k_{m}=$ $(\mathrm{m}+1) \pi / \mathrm{W}_{\mathrm{a}} ; \mathrm{W}_{\mathrm{a}} / \mathrm{W} \rightarrow 1 . \quad \beta_{\mathrm{m}}-\beta_{0}=\pi\left(\mathrm{m}^{2}+2 \mathrm{~m}\right) / 6$ (for a 3-dB coupler) and $\left|\tau_{\mathrm{m}}\right|=1$ for the ideal case. 


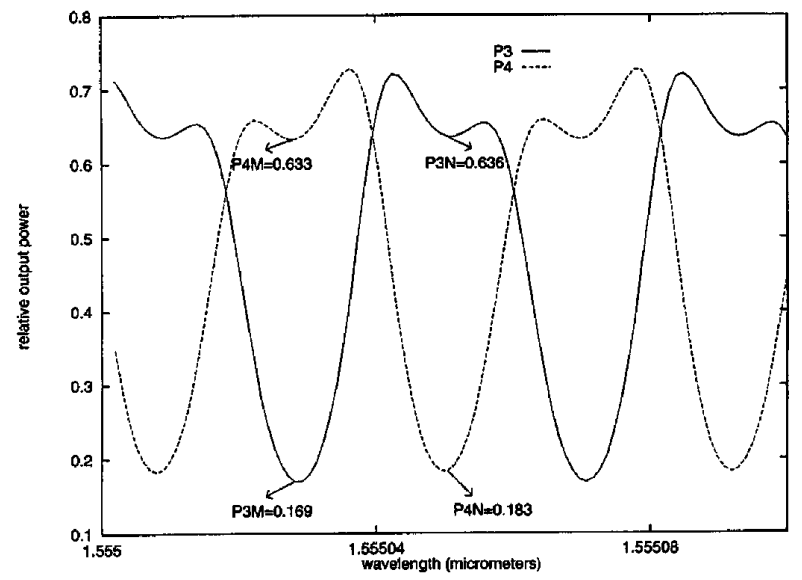

Fig. 12. Calculated output power at ports 3 and 4 as a function of wavelength of a MMI coupler with $\mathrm{W}=12 \mu \mathrm{m}, \mathrm{L}_{\mathrm{MMI}}=225 \mu \mathrm{m}$, $r_{1} r_{2}=0.35$.

After doing so, the foll lowing equations are derived:

$$
\begin{array}{r}
A=\frac{1}{2 W_{a}} \exp \left(i \beta_{0} l\right) \sum_{m=0}^{M} \exp \left[-i \frac{\pi}{6}\left(m^{2}+2 m\right)\right] \\
m \neq 2,5,8 \ldots, \\
B=\frac{1}{2 W_{a}} \exp \left(i \beta_{0} l\right) \sum_{m=0}^{M} \exp \left[-i \frac{\pi}{6}\left(m^{2}+2 m\right)\right](-1)^{m+1} \\
m \neq 2,5,8 \ldots \text { (A8) }
\end{array}
$$

The aim when calculating these expressions was to show the capability of applying Eqs. (A2) to MMI couplers, with $\xi$ as a function of the phase shift between $A$ and $B$ in Eqs. (A8). So our analytical calculation procedure can be used.

This has also been demonstrated in a more precise way. From a multimode analysis ${ }^{8}$ the coefficients A and $B$ are cal culated. These values are used in Eqs. (1) with $\Delta \Phi_{1}=\Delta \Phi_{2}=0$, and from the extremes of the output powers we determine the loss and the powertransfer ratio with Eqs. (8) and (9).

Figure 12 shows the FP resonance that has been obtained for a MMI coupler designed in a InGaAsP buried-type waveguide on InP substrate with an effective index $N_{1}=3.232$ for a TE input beam at 1.55 $\mu \mathrm{m}$. Other parameters are $\mathrm{W}=12 \mu \mathrm{m}, \mathrm{L}_{\mathrm{MMI}}=225$ $\mu \mathrm{m}, r_{1} r_{2}=0.35$, and length of the input-output waveguides $\mathrm{I}=0.85 \mathrm{~cm}$.

Using the extremes of Fig. 12 and $\mathrm{Eqs}$. (8) and (9), we obtain $\mathrm{K}_{\mathrm{r}}=0.49, \mathrm{G}=0.52 \mathrm{~dB}$ in perfect accordance with the simulation when the resonance is not considered. This last result is also corroborated through the (beam-propagation method).

The small deviation from the ideal behavior of the coupler, a phase difference of $88^{\circ}$ between output ports, results in the slight asymmetry of Fig. 12.

We thank Angel Ferreiro and Francisco J. Mustieles for their helpful discussions. This work was supported by a Grant from the Spanish Ministry of Education and Science.

\section{References}

1. M. D. Feit, J . A. Fleck, and L. McCaughan, "Comparison of calculated and measured performance of diffused channelwaveguide couplers,"J . Opt. Soc. Am. 73, 1296-1304(1983).

2. K. Kishioka and G. L. Yip, "Simple measurement technique for the coupling coefficient of integrated optic directional couplers," Appl. Opt. 15, 2807-2813(1992).

3. W. J . Tomlinson, A. Shahar, and R. J . Deri, "Use and misuse of end-facet reflections in the characterization of optical waveguides directional couplers,"Appl. Opt. 30, 2961-2969 (1991).

4. R. G. Walker, "Simple and accurate loss measurement technique for semiconductor optical waveguides,"Electron. Lett. 13, 581-583 (1985).

5. K. Al-hemyari, G. F. Doughty, C. D. W. Wilkinson, A. H. Kean, and C. R. Stanley, "Optical loss measurement on Ga As/GaAlAs single-mode waveguide y-junctions and waveguide bends," J . Lightwave Technol. 11, 272-276 (1993).

6. R. Ulrich and T. Kamiya, "Resolution of self-images in planar optical waveguides,"J . Opt. Soc. Am. 68, 583-593 (1978).

7. L. S. Yu, Q. Z. Liu, S. A. Pappert, and S. S. Lau, "Laser spectral linewidth dependence on waveguide loss measurements using the Fabry-Perot method," Appl. Phys. Lett. 64, 536-538 (1994).

8. C. Vázquez, F. J . Mustieles, and F. Hernández Gil, "Modelado tridimensional mediante el ementos finitos de acopladores interferenciales multimodo," in Union Cientifica Internacional de Radio '94 (Universidad de Las Palmas de Gran Canaria, Las Palmas, Canary Islands, Spain, 1994), pp. 898-902. 